\title{
8. Mitgliedschaften in Religionsgemeinschaften, in Vereinen und gemeinnützigen Organisationen und freiwilliges Engagement
}

\author{
Claudia Vogel \& Nicole Hameister
}

\section{Kernaussagen}

Die Anteile freiwillig Engagierter unterscheiden sich deutlich nach Konfessionszugehörigkeit. Mitglieder der Katholischen Kirche und der Evangelischen Kirchen sind anteilig überdurchschnittlich häufig engagiert. Mitglieder Islamischer Religionsgemeinschaften sind wie Menschen ohne Konfessionszugehörigkeit zu geringeren Anteilen freiwillig engagiert.

Mitglied in einem Verein oder einer gemeinnützigen Organisation sind 44,8 Prozent der in Deutschland lebenden Menschen im Alter ab 14 Jahren. Männer, Ältere und hochgebildete Personen sind häufiger Mitglied als Frauen, Jüngere und niedriggebildete Personen.

Der Anteil von Personen, die Mitglied in einem Verein oder einer gemeinnützigen Organisation sind, ist 2014 deutlich höher als 2009. Der Anstieg beläuft sich von insgesamt 37,8 Prozent auf 44,8 Prozent. Da der Anteil bei Frauen, aber auch bei Männern gestiegen ist, sind Frauen mit einem Anteil von 40,2 Prozent nach wie vor seltener Mitglied als Männer (49,5 Prozent).

Bei der Altersgruppe der 14- bis 29-Jährigen ist der Anteil der Mitglieder in Vereinen oder gemeinnützigen Organisationen nach wie vor am geringsten. Allerdings ist der Anstieg in dieser Altersgruppe am stärksten ausgefallen (von 27,9 Prozent im Jahr 2009 auf 41,8 Prozent im Jahr 2014), sodass sich die Anteile über die Altersgruppen angeglichen haben.

Hochgebildete sind deutlich häufiger Mitglied in einem Verein oder einer gemeinnützigen Organisation als Niedriggebildete. Außerdem sind Schülerinnen und Schüler überdurchschnittlich häufig Mitglieder, und bei dieser Gruppe ist der Anstieg besonders hoch (von 30,8 Prozent im Jahr 2009 auf 50,6 Prozent im Jahr 2014).

Vereine und gemeinnützige Organisationen bieten Gelegenheiten, sich freiwillig zu engagieren. Mitglieder engagieren sich zu deutlich höheren Anteilen freiwillig als Personen, die in keinem Verein und in keiner gemeinnützigen Organisation Mitglied sind.

\subsection{Einleitung}

Freiwillig Engagierte sind in der Regel für zivilgesellschaftliche Organisationen tätig, hierzu zählen zum Beispiel Vereine und andere gemeinnützige Organisationen, oder für Einrichtungen, die etwa von Kirchen oder religiösen
Vereinigungen betrieben werden, wenn sie nicht staatlich oder kommunal gefördert sind (siehe Kapitel 20). Für diese Art des organisationsgebundenen und formal organisierten freiwilligen Engagements ist anzunehmen, dass das Merk-

(C) Der/die Autor(en) 2017

J. Simonson et al. (Hrsg.), Freiwilliges Engagement in

Deutschland, Empirische Studien zum bürgerschaftlichen

Engagement, DOI 10.1007/978-3-658-12644-5_9 
mal der formalen Mitgliedschaft von Bedeutung ist für die Entscheidung der Einzelnen, sich zu engagieren oder nicht.

Erstens bringt die Mitgliedschaft in einer Kirche oder Religionsgemeinschaft Möglichkeiten zum freiwilligen Engagement mit sich (Ehrhardt 2011; Seidelmann 2012; Coenen-Marx 2011). Kirchen und religiöse Organisationen sind in allen Gemeinden Deutschlands direkt vor Ort tätig und nehmen neben den religiösen Aufgaben auch häufig karitative oder edukative Aufgaben wahr, für deren Bewältigung sie teils auf ehrenamtliche Kräfte zurückgreifen, die sie aus ihren Reihen anwerben. Zweitens kann davon ausgegangen werden, dass sich Personen mit Konfessionszugehörigkeit von Personen ohne Konfessionszugehörigkeit unterscheiden, etwa wenn sie unterschiedliche Werte und Weltanschauungen vertreten. Viele Religionsgemeinschaften sind jedoch nicht als Vereine organisiert, denn sie besitzen den Status einer Körperschaft des Öffentlichen Rechts. ${ }^{1} \mathrm{Zu}$ den Religionsgemeinschaften mit einem solchen Status zählen in Berlin neben der Evangelische Kirche und der Katholischen Kirche etwa die Jüdische Gemeinde, die Neuapostolische Kirche sowie die Zeugen Jehovas. Islamischen Organisationen blieb die Rechtsform einer Körperschaft des Öffentlichen Rechts bislang verwehrt. Muslime in Deutschland organisieren sich deshalb in religiösen Vereinen (Kandel 2004). Allerdings werden nur ein kleiner Teil der rund vier Millionen Muslime, die in Deutschland leben, von entsprechenden muslimischen Organisationen vertreten (Haug, Müssig \& Stichs 2009). Wir möchten im Folgenden für die drei größten Konfessionsgruppen in Deutschland das sind mit Abstand die Katholische Kirche und die Evangelische Kirche mit den Evangelischen Freikirchen sowie die weniger verbreitete Reli-

1 Eine Übersicht über alle Religionsgemeinschaften, die in den 16 Ländern jeweils den Status einer Körperschaft des Öffentlichen Rechts besitzen, wird vom Bundesministerium des Innern (BMI 2016) bereitgestellt. gionsgemeinschaft des Islam $^{2}$ - darstellen, wie hoch die jeweiligen Anteile freiwillig Engagierter sind und wie sich diese unterscheiden vom Anteil der Personen ohne Konfessionszugehörigkeit sowie mit sonstiger Konfessionszugehörigkeit.

Auch die Mitgliedschaft in einem Verein bietet Gelegenheiten, sich freiwillig zu engagieren. Die Vereinsmitgliedschaft beinhaltet in der Regel Mitgliedschaftsrechte, etwa die Vereinsangebote $\mathrm{zu}$ nutzen und in der Mitgliederversammlung abzustimmen, aber meist auch Pflichten, etwa einen Mitgliedsbeitrag zu bezahlen und Aufgaben im Rahmen der Vereinstätigkeit zu übernehmen - bei Mitgliedern, die lediglich ihren Beitrag zahlen, spricht man auch von passiver Mitgliedschaft, im Unterschied zu aktiven Mitgliedern, die sich darüber hinaus persönlich einbringen. Die Mitgliedschaft im Verein kann als Vorform des freiwilligen Engagements gesehen werden, da sich aus ihr die Übernahme freiwilliger Aufgaben und Arbeiten im Verein ergeben kann. Natürlich sind nicht alle Vereinsmitglieder freiwillig engagiert, und nicht alle freiwillig Engagierten sind Mitglied in einem Verein. Der Unterschied zwischen der aktiven Mitgliedschaft im Sinne des Mitmachens an gemeinschaftlichen Aktivitäten und dem freiwilligen Engagement im Verein ist ein gradueller. So spielt zum Beispiel jemand ein paar Jahre Fußball im Verein und übernimmt dann auch die Aufgabe, die Jugendmannschaft zu trainieren. Für bestimmte Felder freiwilligen Engagements sind Mitgliedschaften als Zugangsvoraussetzung sogar unabdingbar, so sind politische Ehrenämter meist an die Mitgliedschaft in einer politischen Vereinigung oder Partei geknüpft.

In Deutschland existieren heute rund 600.000 zivilgesellschaftliche Organisationen, mehr als je zuvor (Krimmer \& Priemer 2013). Für das

2 Je nach Datenquelle variieren die Anteile der verschiedenen Religionsgruppen etwas, nach den christlichen Gruppen der Katholiken und Protestanten stellen die Muslime jedoch jeweils die größte nichtchristliche Gruppe dar (Bundeszentrale für politische Bildung 2012). 
Jahr 2008 spricht Adloff (2013) noch von rund 550.000 Vereinen, die in circa 600 dezentralen örtlichen Vereinsregistern der Amtsgerichte eingetragen sind. Bei eingetragenen Vereinen handelt es sich um gemeinnützige Vereine, die keinen wirtschaftlichen Zweck verfolgen. Weitere gemeinnützige Organisationen sind gemeinnützige Gesellschaften mit beschränkter Haftung (gGmbH) oder gemeinnützige Aktiengesellschaften. Die Zahl der Vereine und Non-Profit-Organisationen scheint in Deutschland schon seit längerem zu wachsen, da stetig neue Organisationen - etwa als Fördervereine im Bildungsbereich - gegründet werden, zudem fand nach der Wiedervereinigung eine Welle von Vereins-Neugründungen statt (Krimmer \& Priemer 2013). Eine Vielzahl von Vereinen existiert zum Beispiel in den Bereichen Sport, Freizeit und Kultur, mit fast 100.000 Vereinen sind die Sportvereine am weitesten verbreitet. Es gibt darüber hinaus zahlreiche Traditions- und Hobbyvereine, sowie soziale, karitative und politische Vereine, oder Vereine für Umwelt, für Naturschutz und für Minderheiten- und Menschenrechte (Freitag \& Stadelmann-Steffen 2011). Parteien sind ebenfalls als Vereine organisiert, allerdings in der Regel nicht als rechtsfähige, eingetragene Vereine (Gabriel, Kunz, Roßteutscher \& van Deth 2002). $\mathrm{Zu}$ den unterschiedlichen Arten von freiwilligen Vereinigungen gehören Selbstzweck-Vereine wie Sportvereine, ideelle Vereine wie Bürgerinitiativen und Selbsthilfe- oder Fremdhilfevereine wie Genossenschaften (Müller-Jentsch 2008). Vereine stehen gleichermaßen für eine Rechtsform und für eine Organisationsform (Zimmer 2011). Laut Bürgerlichem Gesetzbuch (BGB) muss ein rechtsfähiger Verein einen Vorstand haben, der den Verein vertritt. Aufgelöst werden können Vereine durch Beschluss der Mitgliederversammlung. Eingetragene Vereine müssen bei ihrer Gründung mindestens sieben Mitglieder haben (Müller-Jentsch 2008). Müller-Jentsch (ebd.) beschreibt Vereine als freiwillige und dauerhafte Zusammenschlüsse von Personen mit gemeinsamen Bedürfnissen und Anliegen. Der Organisationszweck überschneidet sich mit den persönlichen Motiven der Personen, die Mitglied in diesem Verein sind:

Die Ressourcen des Vereins werden hauptsächlich durch Mitgliedsbeiträge und unbezahlte Arbeit der Mitglieder aufgebracht, zum geringeren Teil durch öffentliche Zuschüsse. Die Gestaltung der Organisationsstruktur und des Vereinslebens unterliegt demokratischen Regeln der Mitsprache sowie dem aktiven und passiven Wahlrecht seiner Mitglieder. Der Verein dient keinem Erwerbszweck, das heißt er verfolgt keine - über Selbst- und Fremdhilfe hinausgehenden - materiellen, ökonomischen Interessen, sondern befriedigt kommunikative, sportliche, musische, kulturelle, politische, religiöse, philanthropische, karitative, gemeinschaftsfördernde etc. Interessen in einem lokal oder regional begrenzten Wirkungsbereich. Der Verein mag zur gemeinsamen Gestaltung der Freizeit, zur Verwirklichung von Ideen oder zur Durchsetzung von Werten, zur Pflege des Brauchtums oder eines künstlerischen Lebenswerkes gegründet worden sein - welche Aufgabe er sich auch immer stellt, er bleibt dabei dem lebensweltlichen Umkreis seiner Mitglieder und deren Bedürfnis nach Geselligkeit und sozialen Kontakten verhaftet. (ebd.: 4f.)

Vereine sind zudem also „Orte der Geselligkeit und der sozialen Integration“, da sie ihren Mitgliedern die Möglichkeit des persönlichen Kontaktes bieten (Adloff 2013: 919). Nicht bei allen, aber bei denjenigen Vereinen, deren Anliegen sich nicht in erster Linie auf die Interessen der eigenen Mitglieder richtet - Beispiele sind unter anderem religiöse und kulturelle Hilfsvereine oder Fördervereine -, „kommt es zu Hilfsbereitschaft, politischem Interesse und generell vertrauens- und integrationsfördernden Effekten“" (ebd.: 919). Allerdings warnt Adloff auch, dass Vereine „weder immer die Grundlage für Demokratie“ sind, noch „,automatisch gesellschaftliche, kulturelle und politische Integration mit sich bringen“ (ebd.: 919). Explizit weist Vortkamp (2008) darauf hin, dass Vereine oft eine homogene Mitgliedschaft haben und gerade dadurch die Exklusion bestimmter Bevölkerungsgruppen 
befördern können. In der historischen Entwicklung vieler Vereine wurden Frauen häufig ausgeschlossen (Agricola 1997). Auch verschiedene Gruppen mit Migrationshintergrund sind in Vereinen häufig unterrepräsentiert, hier müssen viele Akteure die interkulturelle Öffnung ihrer Organisation erst noch vorantreiben (Ilgün-Birhimeoglu 2014).

Aus der Literatur ist bekannt, dass sich Vereinsmitglieder mit größerer Wahrscheinlichkeit freiwillig engagieren als Personen, die in keinem Verein Mitglied sind. Wilson und Musick (1998) verweisen auf eine Reihe von Veröffentlichungen, welche die Bedeutung von Mitgliedschaft als Voraussetzung für freiwilliges Engagement belegen. Durch das Mitmachen in Vereinen ergeben sich nicht selten erst Anlässe wie eine Feier oder eine Wettkampfveranstaltung, beziehungsweise Gelegenheiten - es werden ein Übungsleiter, ein Platzwart oder eine Schriftführerin gesucht -, zu helfen und sich freiwillig zu engagieren. Vereine als zivilgesellschaftliche Akteure verbessern somit die Opportunitätsstrukturen für freiwilliges Engagement (siehe Kapitel 20). Verba, Schlozman \& Brady (1995) sprechen deshalb von einem mobilisierenden Effekt der freiwilligen Vereinigungen. In Vereinen erhalten Personen Einblick in soziale Zusammenhänge und werden sich ihrer sozialen Verantwortung, aber auch ihrer Gestaltungsmöglichkeiten bewusst. Zudem zeigt sich empirisch nicht nur, dass sich Vereinsmitglieder etwa in einem Sportverein zu einem höheren Anteil freiwillig engagieren als Personen, die nicht Mitglied sind, sondern zudem, dass sie sich mit zunehmender Mitgliedschaftsdauer auch häufiger engagieren (Schlesinger \& Nagel 2013).

Darüber hinaus gibt es empirische Hinweise darauf, dass Vereinsmitglieder sich durch ein höheres politisches Interesse sowie eine größere Toleranz und weniger Fremdenfeindlichkeit ausweisen als Personen, die nicht in einem Verein sind (Braun \& Hansen 2004). Die Mitgliedschaft in Vereinen muss dafür aber nicht unbedingt die Ursache sein, es könnte einfach sein, dass das Mitmachen in Vereinen in der Regel eher für Menschen attraktiv ist, die von Vornherein bereits sozialer und toleranter eingestellt sind. Es ist auch zu erwarten, dass Personen mit hoher Bildung häufiger Mitglied in einem Verein sind als Personen mit niedriger Bildung, da eine bessere individuelle Ressourcenausstattung es erleichtert, seine Interessen zu artikulieren und zu organisieren (siehe Kapitel 9). Finden wir hingegen, dass Personen mit geringer und mit hoher Bildung gleichermaßen Mitglied im Verein sind, könnte zum Beispiel die Heterogenität im Verein gegenseitige Toleranz durch gegenseitiges Kennenlernen fördern - zwischen Bildungsgruppen, oder zwischen Gruppen unterschiedlicher Herkunft -, jedoch nur dann, wenn die Personen sich tatsächlich treffen und austauschen, das heißt im selben Verein Mitglied sind statt in unterschiedlichen Vereinen.

Der Anteil an Mitgliedern in Vereinen und gemeinnützigen Organisationen wird auch als Indikator für den sozialen Zusammenhalt in der Gesellschaft betrachtet. In der Literatur wird jedoch debattiert, dass in postindustriellen Gesellschaften, die durch Individualisierung gekennzeichnet sind, weniger Personen Mitglied in freiwilligen Vereinigungen werden als in industriellen Gesellschaften (Dekker \& Van den Broek 1998). Große Mitgliederorganisationen wie die Gewerkschaften, die traditionell auch als Vereine organisiert sind, verlieren Mitglieder und berichten auch in Deutschland in regelmäßigen Abständen davon, wie schwierig die Mobilisierung und Neugewinnung von Mitgliedern ist (Offe \& Fuchs 2001; Zimmer 2013). Gleichzeitig entstehen mehr und mehr neu gegründete Vereine, zum Beispiel Fördervereine für Schulen, es wäre also naheliegend anzunehmen, dass dadurch insgesamt mehr Personen Mitglieder werden. Allerdings wird sich eine wachsende Zahl von Vereinen nur dann in einer Zunahme des Mitgliederanteils in der Bevölkerung niederschlagen, wenn diese neuen Vereine auch eine große Zahl von Mitgliedern ansprechen und wenn in diesen neuen Vereinen auch Personen Mitglieder werden, die vorher noch in keinem anderen Verein Mitglied waren. 
In diesem Kapitel soll folgenden vier Forschungsfragen nachgegangen werden:

1. Welche Unterschiede zeigen sich in den Anteilen Engagierter nach der Zugehörigkeit zu einer Religionsgemeinschaft?

2. Wie hoch ist der Anteil von Personen insgesamt und in verschiedenen Bevölkerungsgruppen, die Mitglied in einem Verein oder einer gemeinnützigen Organisation sind?

3. Wie hat sich dieser Anteil von 2009 auf 2014 verändert? Steigt oder sinkt also der Anteil von Mitgliedern in gemeinnützigen Organisationen und Vereinen an der Bevölkerung im Zeitverlauf? Die Entwicklung des Anteils der Mitglieder ist insbesondere vor dem Hintergrund zu beschreiben, dass sich durch diese Entwicklung auch die Opportunitätsstrukturen für freiwilliges Engagement verbessern oder aber verschlechtern.

4. Engagieren sich Personen, die Mitglied sind, zu höheren Anteilen als Personen, die kein Mitglied sind?

\subsection{Datengrundlage}

Die in diesem Kapitel berichteten Befunde basieren auf gewichteten Analysen. Für die Gewichtung wurden in allen Erhebungsjahren dieselben Gewichtungsmerkmale berücksichtigt. Dabei handelt es sich um die Designgewichtung, die sich auf die Auswahlwahrscheinlichkeit einer Person bezieht (Haushaltsgröße, Zahl der Festnetznummern und, nur für das Jahr 2014, auch die Zahl der Mobilfunknummern) und Gewichtungsmerkmale, die Abweichungen der Stichprobenverteilung von der Grundgesamtheit in bestimmten Dimensionen ausgleichen (Bundesland, Gemeindegrößenklassen, Geschlecht, Altersgruppen). Von einer Hinzunahme weiterer Gewichtungsmerkmale wie etwa des Bildungsstandes haben wir abgesehen, um die Vergleichbarkeit mit den bisher veröffentlichten Berichten zu erhalten.

Im Freiwilligensurvey 2014 wird nach der Religionszugehörigkeit gefragt:

Gehören Sie einer Konfession oder Religionsgemeinschaft an?

Wenn ja:
Um welche Konfession oder Religionsgemeinschaft handelt es sich?

Wir betrachten im Folgenden fünf Kategorien: die Katholische Kirche (Antwortkategorie ,römisch-katholische Kirche'), die Evangelischen Kirchen (Antwortkategorien ,evangelische Kirche' und ,evangelische Freikirchen'), die Islamischen Religionsgemeinschaften (Antwortkategorie ,islamische Religionsgemeinschaft'), sonstige Konfessionszugehörigkeit (Antwortkategorien ,Judentum', ,Christlich-Orthodoxe Kirche, ,Neuapostolische Kirche‘, ,Zeugen Jehovas, ,Buddhismus', ,Hinduismus' und ,Sonstiges') sowie ,keine Konfessionszugehörigkeit‘.

Während Frauen sowohl in der Katholischen Kirche als auch in den Evangelischen Kirchen einen höheren Anteil stellen als Männer, ist der Anteil Konfessionsloser bei den Männern größer als bei den Frauen (Tabelle 8-1). Die Islamischen Religionsgemeinschaften zeichnen sich insbesondere durch den überdurchschnittlichen Anteil bei Schülerinnen und Schülern (8,5 Prozent zu 2,6 Prozent in der Wohnbevölkerung ab 14 Jahren insgesamt) aus. Muslime sind im Vergleich zu den Angehörigen anderer Religionsgemeinschaften vergleichsweise jünger, denn in 
der Altersgruppe der 14- bis 29-Jährigen stellen sie laut unseren Ergebnissen einen Anteil von 5,9 Prozent, in der Altersgruppe der 65-Jährigen und Älteren jedoch lediglich einen Anteil von 0,2 Prozent (Tabelle 8-1).

Tabelle 8-1: Konfessionszugehörigkeit nach Geschlecht, Alter und Bildung, 2014

\begin{tabular}{lccccc}
\hline & $\begin{array}{c}\text { Katholische } \\
\text { Kirche }\end{array}$ & $\begin{array}{c}\text { Evangelische } \\
\text { Kirchen und } \\
\text { Freikirchen }\end{array}$ & $\begin{array}{c}\text { Islamische } \\
\text { Religionsge- } \\
\text { meinschaften }\end{array}$ & $\begin{array}{c}\text { Sonstige } \\
\text { Konfessions- } \\
\text { zugehörigkeit }\end{array}$ & $\begin{array}{c}\text { Keine } \\
\text { Konfessions- } \\
\text { zugehörigkeit }\end{array}$ \\
\hline Gesamt & 28,0 & 30,4 & 2,6 & 2,5 & 36,6 \\
\hline Frauen & 29,5 & 32,5 & 2,2 & 2,8 & 33,1 \\
\hline Männer & 26,4 & 28,2 & 3,0 & 2,1 & 40,2 \\
\hline 14-29 Jahre & 27,3 & 28,5 & 5,9 & 2,7 & 35,6 \\
\hline 30-49 Jahre & 27,9 & 26,5 & 3,7 & 3,1 & 38,9 \\
\hline 50-64 Jahre & 27,7 & 29,4 & 0,7 & 2,1 & 40,1 \\
\hline 65 Jahre und älter & 28,9 & 38,2 & 0,2 & 2,0 & 30,6 \\
\hline Noch Schüler/in & 27,8 & 29,2 & 8,5 & 3,9 & 30,6 \\
\hline Niedrige Bildung & 33,8 & 30,8 & 2,6 & 2,0 & 30,9 \\
\hline Mittlere Bildung & 24,6 & 29,6 & 2,3 & 2,1 & 41,4 \\
\hline Hohe Bildung & 27,5 & 30,9 & 2,2 & 2,9 & 36,6 \\
\hline
\end{tabular}

Quelle: FWS 2014, gewichtet, eigene Berechnungen (DZA). Basis: Alle Befragten ( $n=28.530$ ).

Dargestellt sind Zeilenprozente (die Prozentzahlen innerhalb einer Zeile summieren sich zu 100 Prozent).

Ob jemand Mitglied in einem Verein ist, wird im Deutschen Freiwilligensurvey 2014 so erhoben:

Sind Sie Mitglied in einem Verein oder einer gemeinnützigen Organisation? Bitte zählen Sie eine Mitgliedschaft in einer Kirche beziehungsweise in einer Religionsgemeinschaft nicht mit dazu.

Im Jahr 2009 war die Frage genauso formuliert, der explizite Hinweis, eine Mitgliedschaft in der Kirche oder einer Religionsgemeinschaft nicht mitzuzählen, fehlte allerdings. Die Anweisung für die Interviewerinnen und Interviewer, die 2009 die Telefoninterviews führten, lautete jedoch ebenfalls, auf Nachfrage der Befragten eine Kirchenmitgliedschaft nicht dazu zu zählen. Aufgrund der Frageformulierung sind auch Mitgliedschaften in gemeinnützigen Organisationen eingeschlossen, die den Begriff Verein nicht im
Namen tragen, beispielsweise Berufsverbände, die sich als eingetragene Vereine organisiert haben, und andere gemeinnützige Organisationen, die nicht als Verein organisiert sind, beispielsweise im Bereich Wohnungsbau (DEGEWO, Deutsche Gesellschaft zur Förderung des Wohnungsbaus, gemeinnützige Aktiengesellschaft). Die Information zur Mitgliedschaft liegt im Freiwilligensurvey 2009 und 2014 vor, nicht aber in früheren Wellen (vgl. Gensicke 2015).

Die Erhebung der Mitgliedschaft im Verein oder einer gemeinnützigen Organisation sowie die Erhebung der Konfession werden im Freiwilligensurvey unabhängig von der Erhebung des freiwilligen Engagements durchgeführt. Für alle Personen liegen Informationen $\mathrm{zu}$ ihrer Mitgliedschaft vor, und zwar unabhängig davon, ob sie sich freiwillig engagieren oder nicht. Das heißt, wir wissen beispielsweise, ob jemand, der 
freiwillig engagiert ist, auch Mitglied ist in einem Verein, es ist allerdings nicht zuzuordnen, ob das freiwillige Engagement innerhalb oder außerhalb dieses Vereines ausgeübt wird. Für eine ausführliche Darstellung der zweistufigen Erhebung von Aktivität (zunächst wird nach dem Mitma- chen in vierzehn gesellschaftlichen Bereichen außerhalb von Beruf und Familie gefragt) und Engagement (anschließend nach zusätzlichen unentgeltlich übernommenen Arbeiten und Aufgaben) siehe Kapitel 3.

\subsection{Konfessionszugehörigkeit und freiwilliges Engagement}

Die Anteile Engagierter unterscheiden sich deutlich nach Konfessionszugehörigkeit. Die Menschen ohne Konfessionszugehörigkeit engagieren sich unterdurchschnittlich, mit 36,2 Prozent. Mitglieder der Katholischen Kirche (48,6 Prozent) und der Evangelischen Kirchen (49,4 Prozent) sind anteilig überdurchschnittlich häufig engagiert (Abbildung 8-1). Mitglieder Islamischer Religionsgemeinschaften sind mit 26,5 Prozent zu deutlich geringeren Anteilen freiwillig engagiert. Diese Ergebnisse stimmen mit Befunden anderer Studien zum Zusammenhang von Religion und Engagement überein, in denen ebenfalls geringere Wahrscheinlichkeiten für Engagement bei Muslimen berichtet werden (Traunmüller 2009). Begründet werden die höheren Quoten der Mitglieder der großen christlichen Kirchen unter anderem durch deren Positionen in der Infrastruktur der Zivilgesellschaft, die zum Beispiel durch wirtschaftliche Stärke (Vorhandensein von Immobilien etc.), aber auch durch Machtpositionen in Entscheidungsgremien zum Ausdruck kommt. Muslimische Vereine sind dagegen bislang weniger vernetzt mit staatlichen, öffentlichen Akteuren und anderen zivilgesellschaftlichen Organisationen.

Darüber hinaus stimmt der Befund einer geringeren Engagementquote von Muslimen überein mit den Ergebnissen, dass sich Personen mit Migrationshintergrund insgesamt zu geringeren Anteilen engagieren als Deutsche ohne Migrationshintergrund, und zwar insbesondere die Personen mit eigener Zuwanderungserfahrung (siehe Kapitel 23). Von den Mitgliedern der Islamischen Religionsgemeinschaften in Deutschland hat in der Tat ein Großteil eine eigene Zuwanderungserfahrung, das heißt, einen Migrationshintergrund der sogenannten ersten Generation. Über die tatsächlichen Ursachen für die vergleichsweise geringe Engagementquote dieser Gruppe lässt sich spekulieren, oft werden neben den bereits genannten strukturellen Gründen auf der individuellen Ebene zum Beispiel mangelnde Deutschkenntnisse oder fehlende Demokratiesozialisation diskutiert, die Ergebnisse sollten jedoch insbesondere bezüglich einer häufig unterstellten religiösen Ursächlichkeit äußerst vorsichtig interpretiert werden.

Nach Geschlecht ist zudem festzuhalten, dass sich Frauen sowohl in der Katholischen Kirche als auch in den Evangelischen Kirchen anteilig seltener freiwillig engagieren als Männer, obwohl Frauen häufiger Kirchenmitglieder sind. Bei Personen mit islamischer Religionszugehörigkeit und bei Personen mit sonstiger Konfessionszugehörigkeit zeigt sich dieser Geschlechterunterschied nicht, hier sind im Gegenteil Frauen anteilig häufiger engagiert als Männer (Abbildung 8-2). Für die Altersgruppen zeigt sich, dass sich die jüngeren Altersgruppen jeweils anteilig häufiger engagieren als die älteste Gruppe der Personen im Alter von 65 und mehr Jahren, wobei die Altersgruppenunterschiede weniger stark ausgeprägt sind bei den Personen ohne Konfessionszugehörigkeit (ohne Abbildung). Dies ist auch ersichtlich an dem hohen Anteil von freiwillig engagierten Schülerinnen und Schülern, etwa in den beiden 
großen christlichen Kirchen. Zudem zeigen sich bei Personen aller Konfessionszugehörigkeiten große Bildungsunterschiede im Anteil Engagierter (ohne Abbildung).

\section{Abbildung 8-1: Anteile Engagierter nach Konfessionszugehörigkeit 2014}

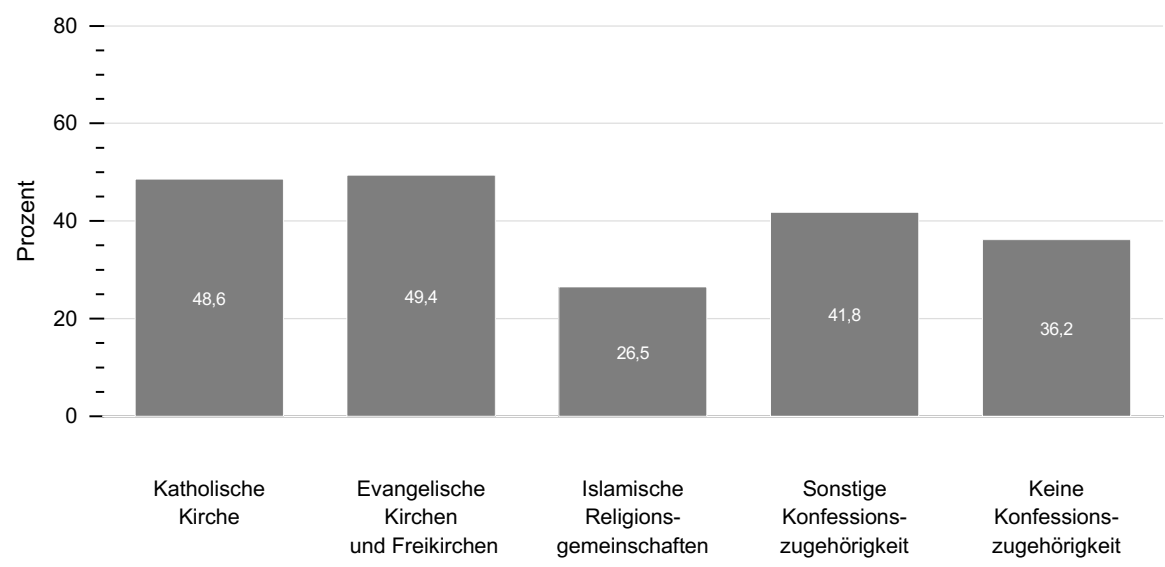

Quelle: FWS 2014, gewichtet, eigene Berechnungen (DZA). Basis: Alle Befragten ( $n=28.529$ ).

\section{Abbildung 8-2: Anteile Engagierter nach Konfessionszugehörigkeit 2014, nach Geschlecht}

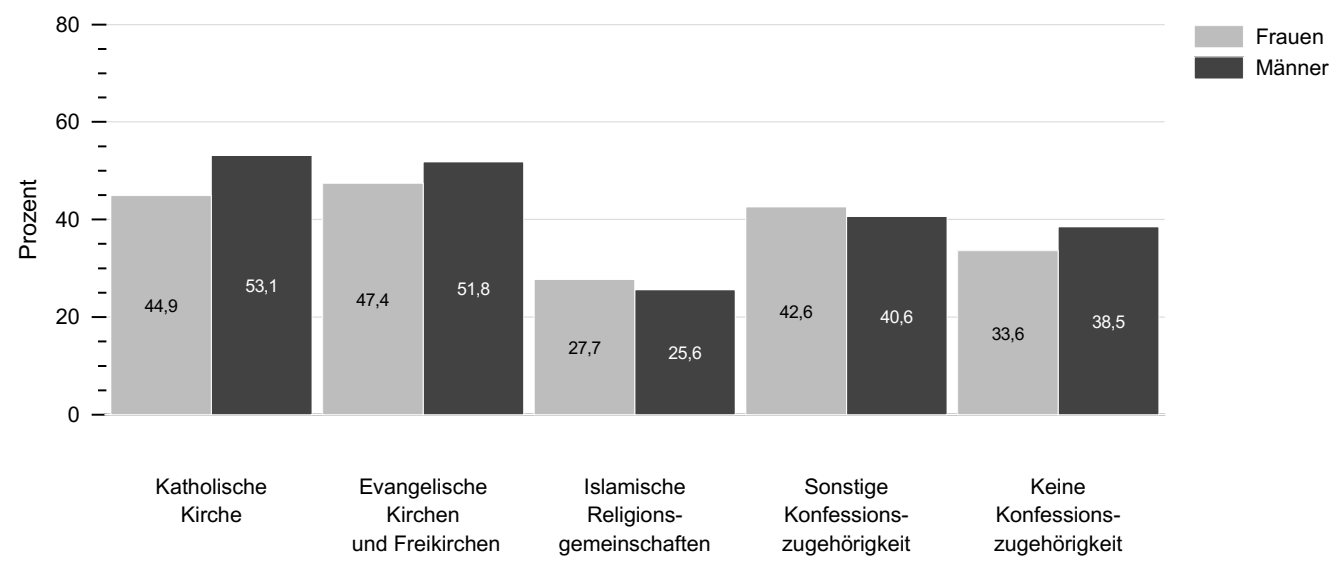

Quelle: FWS 2014, gewichtet, eigene Berechnungen (DZA). Basis: Alle Befragten ( $n=28.529)$. 


\subsection{Mitgliedschaften in Vereinen oder gemeinnützigen Organisationen}

\subsubsection{Anteile der Personen, die 2014 Mitglied sind}

Im Jahr 2014 sind 44,8 Prozent der Wohnbevölkerung Deutschlands im Alter ab 14 Jahren Mitglied in mindestens einem Verein (Abbildung 8-3a). Bei Frauen ist der Anteil von Vereinsmitgliedern etwas niedriger als bei Männern, rund vier von zehn Frauen sind Mitglied in einem Verein und rund fünf von zehn Männern. Es scheint also so zu sein, dass es bei der Vielzahl von Vereinen historisch nach wie vor mehr Vereine gibt, die eher Männer als Frauen ansprechen, also zum Beispiel mehr Fußballvereine, in denen es lediglich Männermannschaften gibt und weniger, in denen es auch Frauenmannschaften gibt, oder mehr Berufsverbände, in denen sich mehr erwerbstätige Männer als erwerbstätige Frauen engagieren. Da Männer nach wie vor zu höheren Anteilen erwerbstätig sind als Frauen, haben sie mehr Gelegenheiten, Mitglied in einem solchen Berufsverband zu sein. Dies wird auch an dem deutlich höheren Anteil gemeinschaftlich aktiver Männer als Frauen im Bereich der beruflichen Interessenvertretung außerhalb des Betriebes deutlich (siehe Kapitel 3). Natürlich existiert heute auch eine Reihe von Vereinen, in denen sich ausschließlich Frauen organisieren, etwa die Landfrauenvereine, in denen sich laut Deutschem LandFrauenverband e.V. allein 500.000 Frauen engagieren (LandFrauen 2014). Die meisten Vereine haben jedoch eine gemischte Mitgliedschaft, also sowohl weibliche als auch männliche Mitglieder.
Die Anteile von Vereinsmitgliedern zwischen den Altersgruppen unterscheiden sich weniger stark, da in Deutschland auch eine Vielzahl von Vereinen existiert, die Personen in jeder Lebenslage und in jedem Lebensalter ansprechen. Allerdings weisen die beiden älteren Altersgruppen mit 46,5 Prozent (bei den Personen im Alter von 65 Jahren und älter) sowie mit 47,6 Prozent (bei den Personen von 50 bis 64 Jahren) etwas höhere Mitgliedsanteile aus als die beiden jüngeren Altersgruppen mit 41,8 Prozent der 14- bis 29-Jährigen und 43,2 Prozent der 30- bis 49-Jährigen (Abbildung 8-3a). Ob dies tatsächlich einen Kohortenunterschied dergestalt darstellt, dass die heute Jüngeren sich weniger für Vereine interessieren als die heute Älteren, oder ob dies ein Lebensphasenunterschied ist, und die Jüngeren später häufiger in Vereinen Mitglied sein werden, wird anhand künftiger empirischer Beobachtungen zu klären sein.

Sehr deutlich ins Gewicht fallen die Unterschiede nach Bildungsabschluss: Während von den Personen mit hoher Bildung mit 50,5 Prozent mehr als die Hälfte Mitglied in mindestens einem Verein ist, sind es bei den Personen mit niedriger Bildung lediglich 37,3 Prozent (Abbildung 8-3b). Darüber hinaus sind Schülerinnen und Schüler überdurchschnittlich häufig in einem Verein Mitglied (50,6 Prozent). Dieser hohe Anteil bei Schülerinnen und Schülern spricht gegen Kohortenunterschiede, weil Jüngere gerade nicht grundsätzlich zu geringeren Anteilen Mitglieder werden. 
Abbildung 8-3: Anteile von Personen, die angeben Mitglied in einem Verein oder einer gemeinnützigen Organisation zu sein, 2014, a) gesamt, nach Geschlecht und nach Alter, b) nach Bildung

a) gesamt, nach Geschlecht und nach Alter

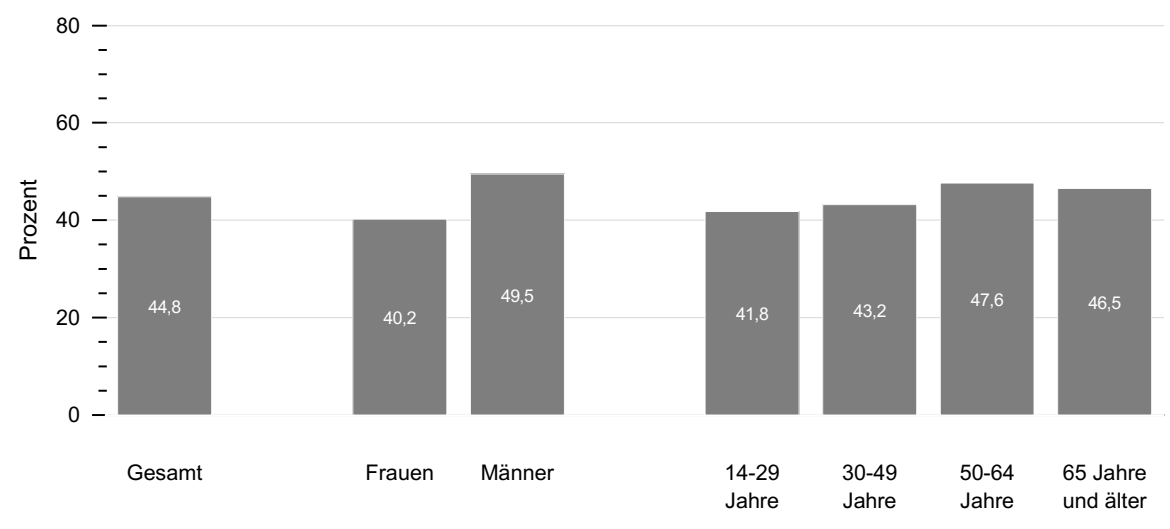

b) nach Bildung

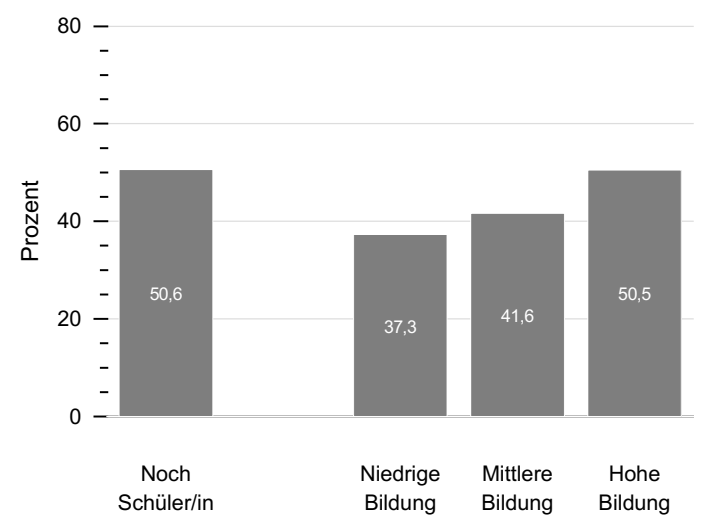

Quelle: FWS 2014, gewichtet, eigene Berechnungen (DZA). Basis: Alle Befragten $(n=28.689)$.

\subsubsection{Anteile der Personen, die Mitglied sind, im Zeitvergleich}

Wie hat sich der Anteil der Personen, die Mitglied in einem Verein sind, im Zeitverlauf entwickelt? Im Jahr 2009, dem ersten Zeitpunkt, zu dem diese Information im Freiwilligensurvey erfasst wurde, waren lediglich 37,8 Prozent der Wohnbevölkerung ab 14 Jahren Mitglied in einem Verein. Der
Anteil von Vereinsmitgliedern ist somit deutlich gestiegen, von 37,8 Prozent auf 44,8 Prozent im Jahr 2014 (Abbildung 8-4).

Dieser Anstieg lässt sich auf den Mitgliederzuwachs bei bestehenden Organisationen wie den Sportvereinen sowie auf die Neugründung von Vereinen zurückführen. Für den Sport ist etwa bekannt, dass die Mitgliederzahlen in den Vereinen von 2000 bis 2010 von knapp 23,4 
Millionen auf rund 23,8 Millionen angestiegen sind (dieser Zuwachs von 410.000 Mitglieder in Sportvereinen entspricht einem Anstieg von knapp zwei Prozent; Fehres, Blessing-Kapelke, Tzschoppe \& Hartmann 2011: 8). Allein der Deutsche Behindertensportverband hat in diesem Zeitraum 226.599 neue Mitglieder gewonnen (ebd.: 23). Der Bereich Sport ist tatsächlich ein Wachstumsbereich, da zunehmend mehr Menschen Sport treiben, sei es präventiv oder zur Rehabilitation. Nach unserem Wissensstand liegen für andere gesellschaftliche Bereiche keine zentralen Information zur Mitgliederentwicklung von Seiten der Organisationen vor. Solche Informationen sind allerdings in Bezug auf das mögliche freiwillige Engagement der Mitglieder auch nur bedingt aussagekräftig, da eine Person in mehreren verschiedenen Vereinen Mitglied sein kann ebenso wie eine Person mehrere freiwillige Tätigkeiten ausüben kann. Im Extremfall könnte es also sein, dass heute gar nicht mehr Personen Mitglied in einem Sportverein sind, sondern viele, die bereits im Jahr 2000 Sport im Verein gemacht haben, nun noch eine weitere Sportart in einem weiteren Verein ausüben. Fest steht aber, dass die Zahl der Vereine wächst und ein großer Teil der Vereine in Deutschland noch recht jung ist - rund zwei Fünftel der Vereine wurden erst nach 1990 gegründet (Alscher, Dathe, Priller \& Speth 2009).

\section{Abbildung 8-4: Anteile von Personen, die angeben Mitglied in einem Verein oder einer gemeinnützigen Organisation zu sein, im Zeitvergleich}

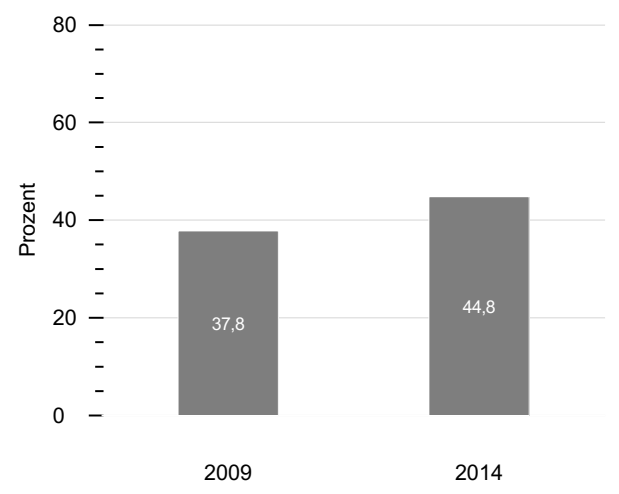

Quelle: FWS, gewichtet, eigene Berechnungen (DZA). Basis: Alle Befragten. FWS 2009 ( $n=20.005$ ), FWS 2014 $(n=28.689)$.

Sowohl Frauen als auch Männer sind heute vergleichsweise häufiger Mitglieder in einem Verein oder einer gemeinnützigen Organisation als 2009 (Abbildung 8-5a). Zudem ist in allen Altersgruppen ein Anstieg des Anteils der Vereinsmitglieder zu verzeichnen, am deutlichsten in der jüngsten Altersgruppe (Abbildung 8-5b). Dieses Ergebnis korrespondiert mit dem Befund $\mathrm{zu}$ den Bildungsgruppen. Am deutlichsten ist der Anstieg des Anteils von Vereinsmitgliedern bei den Schülerinnen und Schülern ausgefallen (Abbildung 8-5c). Dies kann Ausdruck einer gezielten Rekrutierungsstrategie sein, etwa dass Vereine gezielter um den Nachwuchs werben und bereits Schülerinnen und Schüler als Mitglieder $\mathrm{zu}$ gewinnen suchen. Außerdem entstehen viele neue Vereine gerade im Bereich Bildung und Wissenschaft - als Fördervereine oder als Bildungsträger -, die auch direkt auf Schülerinnen und Schüler als Zielgruppe zugeschnitten sind. 
Abbildung 8-5: Anteile von Personen, die angeben Mitglied in einem Verein oder einer gemeinnützigen Organisation zu sein, im Zeitvergleich, a) nach Geschlecht, b) nach Alter, c) nach Bildung

a) nach Geschlecht

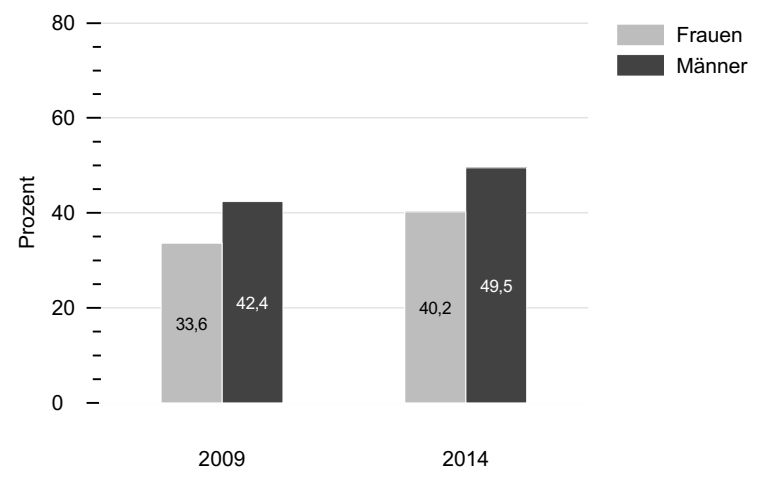

b) nach Alter

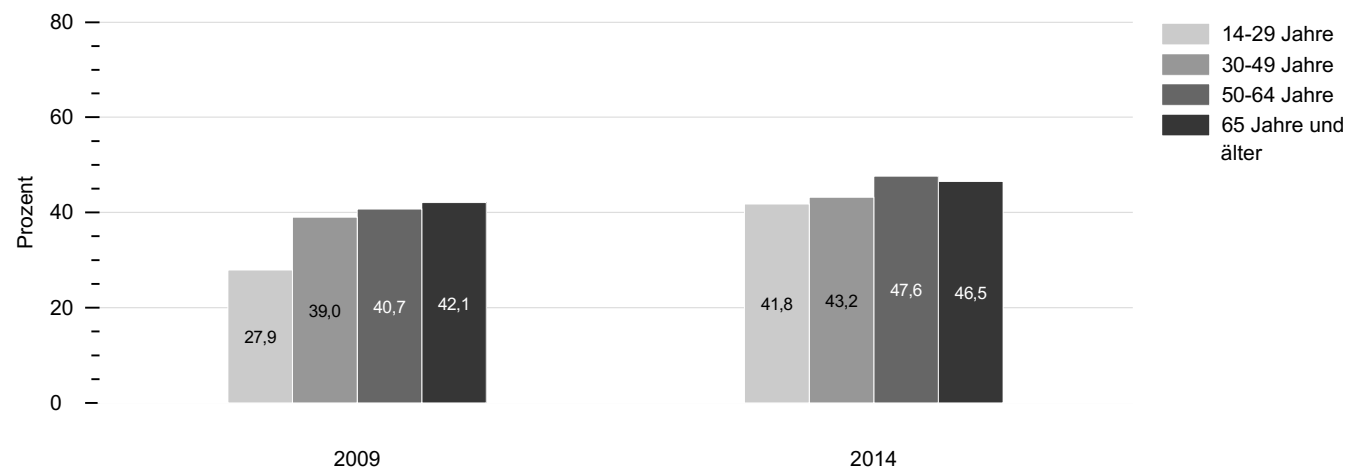

c) nach Bildung

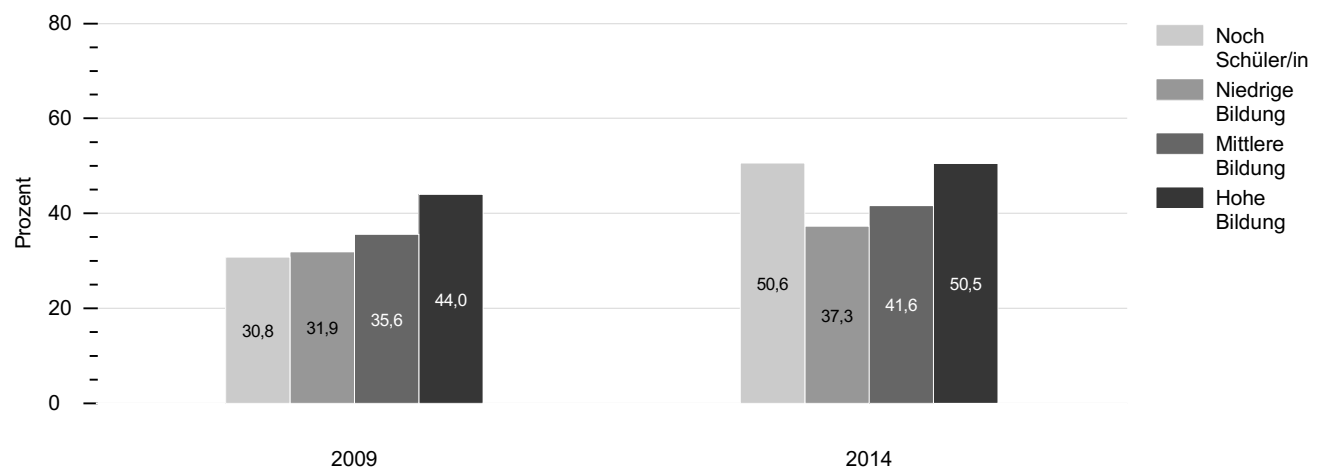

Quelle: FWS, gewichtet, eigene Berechnungen (DZA). Basis: Alle Befragten. FWS 2009 ( $n=20.005)$, FWS 2014 $(n=28.689)$. 


\subsection{Mitgliedschaften in Vereinen oder gemeinnützigen Organisationen und freiwilliges Engagement}

Da die Mitgliedschaft in einem Verein auch als Bedingung für bestimmte vereinsbasierte Formen des freiwilligen Engagements betrachtet werden kann, geht mit dem Anstieg des Anteils der Vereinsmitglieder wohl auch ein Anstieg des Anteils freiwillig Engagierter einher (siehe Kapitel 3). Durch den gestiegenen Mitgliederan- teil an der Bevölkerung haben sich die Opportunitätsstrukturen für freiwilliges Engagement verbessert. Es ist anzunehmen, dass Personen sich meist dort engagieren, wo sie auch Mitglied sind, zudem sind sicherlich einige Vereinsmitglieder in mehreren Organisationen gleichzeitig Mitglied.

Abbildung 8-6: Anteile Engagierter nach Mitgliedschaft 2014, a) gesamt, nach Geschlecht und nach Alter, b) nach Bildung

a) gesamt, nach Geschlecht und nach Alter

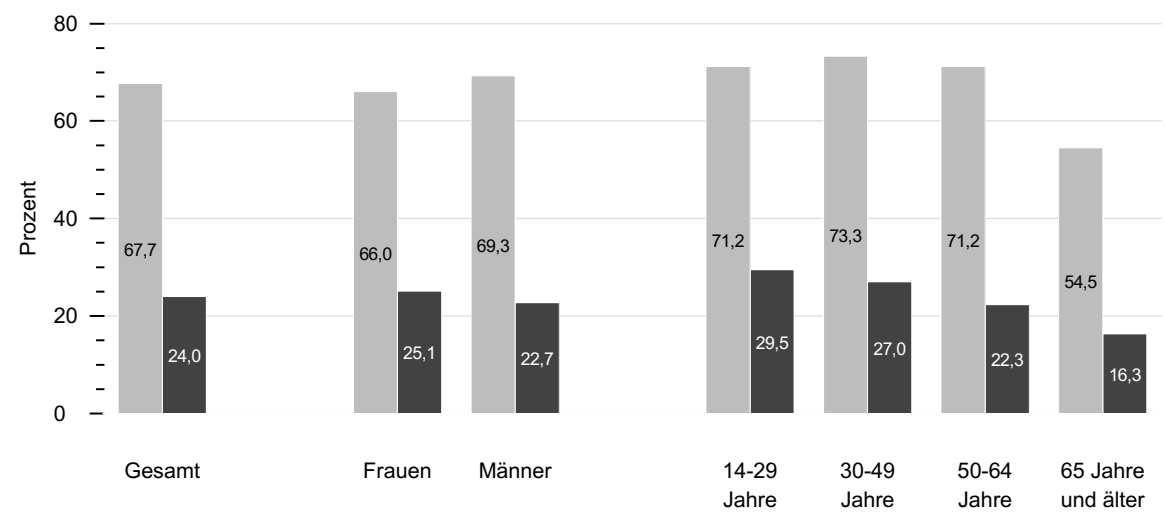

b) nach Bildung

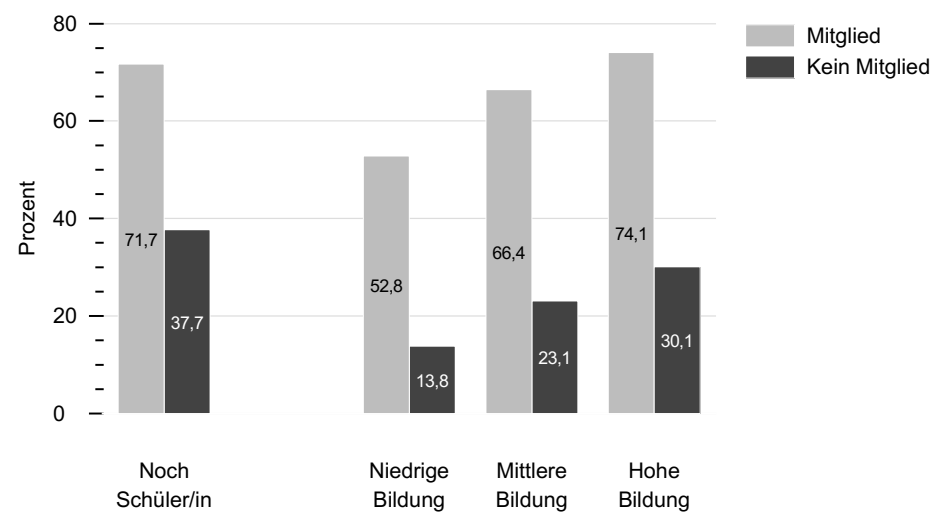

Quelle: FWS 2014, gewichtet, eigene Berechnungen (DZA). Basis: Alle Befragten ( $n=28.689$ ). 
Tatsächlich hängen Mitgliedschaft und freiwilliges Engagement sehr stark zusammen: Mitglieder engagieren sich zu deutlich höheren Anteilen freiwillig als Personen, die in keinem Verein und keiner gemeinnützigen Organisation Mitglied sind. Gut zwei Drittel der Mitglieder sind engagiert, aber weniger als ein Viertel der Nicht-Mitglieder (Abbildung 8-6a). Der Zusammenhang ist für Männer noch deutlicher ausgeprägt als für Frauen. Zwischen den Altersgruppen zeigen sich eher moderate Unterschiede im Zusammenhang von Mitgliedschaft und Engagement, die 65-Jährigen und Älteren sind jedoch deutlich seltener

\subsection{Fazit}

Freiwilliges Engagement wird gefördert und gestützt durch die Organisationen und Einrichtungen, in denen sich Menschen einbringen können. Dies zeigt sich eindrücklich daran, dass sich Mitglieder zu deutlich höheren Anteilen freiwillig engagieren als Personen, die in keinem Verein und keiner gemeinnützigen Organisation Mitglied sind. Auch die Mitglieder der großen christlichen Kirchen - der Katholischen Kirche und der Evangelischen Kirchen - engagieren sich anteilig häufiger freiwillig als Konfessionslose, dies gilt für alle Bevölkerungsgruppen in ähnlichem Maße. Die Anteile freiwillig Engagierter unterscheiden sich somit deutlich nach Mitgliedschaft, aber auch sehr deutlich nach Konfessionszugehörigkeit. Mitglieder Islamischer Religionsgemeinschaften sind in Deutschland $\mathrm{zu}$ geringeren Anteilen freiwillig engagiert als der Durchschnitt der Bevölkerung, wobei die Gründe dafür sicherlich weniger in der Religion selbst als vielmehr in den unterschiedlichen Rahmenbedingungen für freiwilliges Engagement von Personen mit Migrationshintergrund und Personen ohne Migrationshintergrund zu suchen sind (siehe Kapitel 23).

Knapp die Hälfte der Wohnbevölkerung ab 14 Jahren ist aktuell Mitglied in einem oder mehreren Vereinen oder anderen gemeinnützigen freiwillig engagiert, sowohl wenn sie Mitglied sind, als auch, wenn sie kein Mitglied sind.

Bei den Schülerinnen und Schülern beträgt die Engagementquote der Mitglieder 71,7 Prozent und die derjenigen, die kein Mitglied sind, lediglich 37,7 Prozent. Bei den Personen mit geringer Bildung ist der Abstand ebenfalls groß, wenn auch auf einem deutlich niedrigeren Niveau (52,8 Prozent zu 13,8 Prozent). Bei den Personen mit hoher Bildung ist der Abstand ebenfalls sehr stark ausgeprägt, mit 74,1 Prozent zu 30,1 Prozent (Abbildung 8-6b).

Organisationen. Männer sind nach wie vor häufiger Mitglied als Frauen. Zudem ist der höchste Anteil bei Personen im mittleren Lebensalter und der geringste Anteil ist bei den Jüngeren $\mathrm{zu}$ beobachten. Die Tatsache, dass der Mitgliedsanteil auch bei den Älteren geringer ist, könnte auf einen Lebenslaufeffekt verweisen (mit mittlerem Alter wird man Mitglied, im höheren Alter gibt man die Mitgliedschaften auf). Für Vereine, die eine ältere Mitgliederstruktur aufweisen, bedeutet das, sie haben beim Austritt der Mitglieder mit zunehmendem Alter einen Generationenwechsel $\mathrm{zu}$ bewältigen und müssen jüngere Neumitglieder anwerben, um ihre Mitgliederstärke zu erhalten. Niedriggebildete Personen sind zudem seltener Mitglied in einem Verein als hochgebildete Personen. Bezüglich der Vereinsmitgliedschaften zeigen sich somit dieselben Unterschiede zwischen den Bevölkerungsgruppen wie bezüglich des freiwilligen Engagements (siehe Kapitel 3).

Der Anteil von Mitgliedern ist seit 2009 angestiegen. Dadurch sind die Opportunitätsstrukturen für Engagement gestiegen. Wenn mehr Menschen Mitglied sind, ist es naheliegend, dass auch mehr Menschen in den Vereinen und Non-Profit-Organisationen zusätzlich unentgeltliche Aufgaben und Arbeiten übernehmen. 
Tatsächlich findet die Mehrheit des freiwilligen Engagements in Deutschland nach wie vor in der Organisationform der Vereine und Verbände statt (siehe Kapitel 20). Allerdings sind engagierte Frauen seltener in Vereinen freiwillig tätig als Männer (siehe Kapitel 20). Wenn Vereine mehr Kräfte für ehrenamtliche und freiwillige Tätigkeiten werben wollen, dann sollten sie sich zunächst stärker für neue Zielgruppen öffnen. In erster Linie bedeutet das, gezielt Frauen als Mitglieder und Engagierte zu werben, es könnten aber auch gezielt andere Bildungsgruppen oder auch andere Herkunftsgruppen sein. Erreicht werden könnten neue Zielgruppen, indem passgenaue Angebote für sie gemacht werden, die gleichermaßen niedrigschwellig und attraktiv sind, das heißt etwa fremdsprachige persönliche Ansprache.

Hinderungsgründe für die Mitgliedschaft in Vereinen könnten in der Höhe der obligatorischen Mitgliedsbeiträge sowie in den teilweise erforderlichen Aufnahmegebühren liegen. Für Sportvereine liegen die monatlichen Mitgliedschaftsbeiträge durchschnittlich deutlich geringer als Beiträge für gewinnorientierte wirtschaftliche Unternehmen wie Fitness-Studios (Hovemann, Horch \& Schubert 2007). Ausnahmen sind sogenannte Spartenvereine mit hohen Mitgliedsbeiträgen (z. B. Golf, Tennis) von durchschnittlich fast 150 Euro monatlich (Wicker 2009). Vereine finanzieren sich zu großen Teilen über die Beiträge ihrer Mitglieder (zudem über staatliche Zuschüsse, Zuschüssen von Sportorganisationen, Einnahmen aus Veranstaltungen und Spenden; Krimmer, Labigne \& Priemer 2015). Außerdem kann auf Mitgliedsbeiträge keinesfalls vollständig verzichtet werden, da sie, als obligatorische Beiträge, die Mitgliedschaft konstituieren. Allerdings wäre es für einkommensschwache
Personen sicherlich ein Anreiz, wenn diese bei eingeschränkten individuellen finanziellen Spielräumen reduziert oder erlassen werden könnten. Insbesondere wenn mehrere Familienangehörige Vereinsmitglieder werden, oder wenn man in mehreren Vereinen Mitglied ist, können die finanziellen Belastungen das Budget von einkommensschwachen Personen übersteigen. Trotz Familienmitgliedschaften, die über 60 Prozent der Sportvereine anbieten, teilweise zusammen mit Familientarifen (Breuer \& Feiler 2014), können diese Kosten sie davon abhalten, Mitglied zu bleiben beziehungsweise erst zu werden. Die Möglichkeit sich im Verein freiwillig zu engagieren, wäre für diese Personen eingeschränkt. Da die Vereine auf die Mitgliedsbeiträge angewiesen sind, wäre eine Senkung oder zeitweise Aussetzung sicherlich dem dauerhaften Verlust eines Vereinsmitgliedes vorzuziehen. Solche Anreize werden von Vereinen auch heute bereits eingesetzt, um den Engagierten eine quasi-finanzielle Anerkennung für ihre Tätigkeiten zu zollen (als Ersatz oder zusätzlich zu Übungsleiterpauschalen). Schließlich werden von den Einnahmen der Vereine nicht nur die Kosten für Sportanlagen und Sportgeräte getragen, sondern auch die für Trainerinnen oder Trainer und Übungsleiterinnen oder Übungsleiter (Hovemann et al. 2007). In der Politik gibt es bereits erste Maßnahmen, diese Hürden zu verringern: So können Kinder, deren Eltern Arbeitslosengeld II beziehen, über das sogenannte Bildungspaket zehn Euro im Monat für den Mitgliedsbeitrag etwa für den Sportoder den Musikverein erhalten (Bundesministerium für Arbeit und Soziales (BMAS) 2015). Die Politik kann hier sicher noch einiges tun, um Vereine und einkommensschwache Menschen zu unterstützen. 


\section{Literatur}

Adloff, F. (2013). Vereine. In: S. Mau \& N. M. Schöneck (Hrsg.) Handwörterbuch zur Gesellschaft Deutschlands (S. 909921). Wiesbaden: Springer VS.

Agricola, S. (1997). Vereinswesen in Deutschland. Eine Expertise im Auftrag des Bundesministeriums für Familie, Senioren, Frauen und Jugend. Stuttgart: Kohlhammer.

Alscher, M., Dathe, D., Priller, E., \& Speth, R. (2009). Bericht zur Lage und zu den Perspektiven des bürgerschaftlichen Engagements in Deutschland. Berlin: Bundesministerium für Familie, Senioren, Frauen und Jugend.

Braun, S., \& Hansen, S. (2004). Soziale und politische Integration durch Vereine? Forschungsjournal Neue Soziale Bewegungen, 17(1), 62-69.

Breuer, C., \& Feiler, S. (2014). Sportvereine in Deutschland - ein Überblick. In: C. Breuer (Hrsg.) Sportentwicklungsbericht 2013/2014. Analyse zur Situation der Sportvereine in Deutschland. Bonn: Sportverlag Strauß.

Bundesministerium des Innern (BMI) (2016). Religions- und Weltanschauungsgemeinschaften. Online: http://www.personenstandsrecht.de/PERS/DE/Themen/Informationen/Religionsgemeinschaften/religionsgemeinschaften_node. html (zuletzt abgerufen 9.2.2016).

Bundesministerium für Arbeit und Soziales (BMAS) (2015). Das Bildungspaket. Mitmachen möglich machen. Berlin: BMAS.

Bundeszentrale für politische Bildung (2012). Religionszugehörigkeit. Online: http://www.bpb.de/nachschlagen/zahlen-und-fakten/soziale-situation-in-deutschland/145148/religionszugehoerigkeit (zuletzt abgerufen 9.2.2016).

Coenen-Marx, C. (2011). Ehrenamtliches Engagement in der Kirche. In: T. Olk \& B. Hartnuß (Hrsg.) Handbuch Bürgerschaftliches Engagement (S. 257-265). Weinheim: Beltz Juventa.

Dekker, P., \& van den Broek, A. (1998). Civil Society in Comparative Perspective. International Journal of Voluntary and Nonprofit Organizations, 9(1), 11-38.

Ehrhardt, J. (2011). Ehrenamt. Formen, Dauer und kulturelle Grundlagen des Engagements. Frankfurt am Main: Campus.

Fehres, K., Blessing-Kapelke, U., Tzschoppe, P., \& Hartmann, S. (2011). Mitgliederentwicklung in Sportvereinen - Bestandserhebungen und demografischer Wandel zwischen den Jahren 2000 und 2010. Frankfurt am Main: Deutscher Olympischer Sportbund.

Freitag, M., \& Stadelmann-Steffen, I. (2011). Die Schweizer Gemeinden und ihre Vereine: Strukturen - Leistungen - Bewertungen. In: H. Ammann (Hrsg.) Grenzen-Los! Fokus Gemeinde. Freiwilliges Engagement in Deutschland, Österreich und der Schweiz. Referate der 2. Internationalen Vernetzungskonferenz 2010 in Rüschlikon (S. 35-66). Zürich: Seismo.

Gabriel, O., Kunz, V., Roßteutscher, S., \& Deth, J. W. van (2002). Sozialkapital und Demokratie. Wien: WUV-Universitätsverlag.

Gensicke, T. (2015). Freiwilliges Engagement in Deutschland: Freiwilligensurvey 2009. Wiesbaden: Springer VS.

Haug, S., Müssig, S., \& Stichs, A. (2009). Muslimisches Leben in Deutschland. Nürnberg: Bundesamt für Migration und Flüchtlinge.

Hovemann, G., Horch, H.-D., \& Schubert, M. (2007). Sportvereine und Finanzen. In: C. Breuer (Hrsg.) Sportentwicklungsbericht 2005/2006. Analyse zur Situation der Sportvereine in Deutschland. Bonn: Sportverlag Strauß.

Ilgün-Birhimeoglu, E. (2014). Interkulturelle Öffnung in Organisationen des ehrenamtlichen Engagements. In: E. Vanderheiden \& C.-H. Meyer (Hrsg.) Handbuch interkulturelle Öffnung (S. 373-382). Göttingen: Vandenhoeck \& Ruprecht.

Kandel, J. (2004). Was ist Is/am? Online: http://www.bpb.de/politik/innenpolitik/konfliktstoff-kopftuch/63315/islamin-deutschland? $\mathrm{p}=0$ (zuletzt abgerufen 5.2.2016).

Krimmer, H., Labigne, A., \& Priemer, J. (2015). Wie finanzieren sich zivilgesellschaftliche Organisationen in Deutschland? Eine Sonderauswertung des ZiviZ-Surveys. Online: http://www.koerber-stiftung.de/gesellschaft/weitere-arbeitsfelder/forum-engagementfoerderung/treffen/hamburg-januar-2015.html (zuletzt abgerufen 5.2.2016).

Krimmer, H., \& Priemer, J. (2013). ZIVIZ-Survey 2012. Zivilgesellschaft verstehen. Berlin: Stifterverband für die Deutsche Wissenschaft.

LandFrauen (2014). Landfrauen mit Ideen. Jahresbericht 2014. Online: http://www.landfrauen.info/fileadmin/Redaktion/ PDF/Publikationen/dlv_Jahresbericht_2014.pdf (zuletzt abgerufen 5.2.2016). 
Müller-Jentsch, W. (2008). Der Verein - Ein blinder Fleck der Organisationssoziologie. Berliner Journal für Soziologie, 18(3), 476-503.

Offe, C., \& Fuchs, S. (2001). Schwund des Sozialkapitals? Der Fall Deutschland. In: R. D. Putnam (Hrsg.) Gesellschaft und Gemeinsinn. Sozialkapital im internationalen Vergleich (S. 417-514). Gütersloh: Bertelsmann Stiftung.

Schlesinger, T., \& Nagel, S. (2013). Who will volunteer? Analysing individual and structural factors of volunteering in Swiss sports clubs. European Journal of Sport Science, 13(6), 707-715.

Seidelmann, S. (2012). Evangelische engagiert - Tendenz steigend. Sonderauswertung des dritten Freiwilligensurveys für die evangelische Kirche. Hannover: Sozialwissenschaftliches Institut der Evangelischen Kirche in Deutschland.

Traunmüller, R. (2009). Religion und Sozialintegration. Eine empirische Analyse der religiösen Grundlagen sozialen Kapitals. Berliner Journal für Soziologie, 3, S. 435-468.

Verba, S., Schlozman, K. L., \& Brady, H. E. (1995). Voice and equality. Civic voluntarism in American politics. Cambridge: Harvard University Press.

Vortkamp, W. (2008). Integration durch Teilhabe. Das zivilgesellschaftliche Potenzial von Vereinen. Frankfurt am Main: Campus.

Wicker, P. (2009). Perspektiven und Grenzen der Beitragsfinanzierung von Sportvereinen - Eine Analyse zur Theorie und Empirie der Beitragselastizität im Sportverein. Köln: Deutsche Sporthochschule Köln.

Wilson, J., \& Musick, M. (1998). The Contribution of Social Resources to Volunteering. Social Science Quarterly, 79(4), 799-814.

Zimmer, A. (2013). Auslaufmodell Verein? Vom Veralten eines gesellschaftlichen Strukturmoments. Theorie und Praxis der sozialen Arbeit, 64(6), 447-455.

Zimmer, A. (2011). Vereine. In: T. Olk \& B. Hartnuß (Hrsg.) Handbuch Bürgerschaftliches Engagement (S. 453-463). Weinheim: Beltz Juventa.

Open Access Dieses Kapitel wird unter der Creative Commons Namensnennung 4.0 International Lizenz (http://creativecommons.org/licenses/by/4.0/deed.de) veröffentlicht, welche die Nutzung, Vervielfältigung, Bearbeitung, Verbreitung und Wiedergabe in jeglichem Medium und Format erlaubt, sofern Sie den/die ursprünglichen Autor(en) und die Quelle ordnungsgemäß nennen, einen Link zur Creative Commons Lizenz beifügen und angeben, ob Änderungen vorgenommen wurden.

Die in diesem Kapitel enthaltenen Bilder und sonstiges Drittmaterial unterliegen ebenfalls der genannten Creative Commons Lizenz, sofern sich aus der Abbildungslegende nichts anderes ergibt. Sofern das betreffende Material nicht unter der genannten Creative Commons Lizenz steht und die betreffende Handlung nicht nach gesetzlichen Vorschriften erlaubt ist, ist für die oben aufgeführten Weiterverwendungen des Materials die Einwilligung des jeweiligen Rechteinhabers einzuholen. 